\title{
La lengua católica de Alberto Liptay ${ }^{1}$
}

\author{
Carmen Galán Rodríguez* \\ Universidad de Extremadura, España
}

\begin{abstract}
Resumen
El objeto de este trabajo es analizar la lengua católica, un sistema artificial a posteriori ideado por el cirujano de la marina chilena Alberto Liptay (1859-1922). El proyecto es un modelo exquisito de racionalidad, y uno de los escasos sistemas en español, pero no tuvo apenas reconocimiento entre sus contemporáneos, quizá porque el autor se declaraba tan solo un filólogo aficionado. Un estudio atento de sus numerosas contribuciones ofrece, sin embargo, lecturas ideológicas muy sugerentes del autor que determinaron en gran medida la configuración de su propuesta lingüística.
\end{abstract}

Palabras clave: Alberto Liptay, lenguas artificiales a posteriori naturalistas, lengua católica, ideología.

1 Este trabajo se ha realizado en el marco del Proyecto de Investigación FFI2016-76702-P, titulado En los límites del lenguaje: diseños artificiales y ficciones comunicativas (Ministerio de Economía y Competitividad), dirigido por la Dra. Carmen Galán Rodríguez.

* Para correspondencia, dirigirse a: Carmen Galán Rodríguez (cgalan@unex.es), U. de Extremadura, Facultad de Filosofía y Letras, Departamento de Filología Hispánica y Lingüística General. Av. de la Universidad, s/n, 10074 Cáceres (España). 


\title{
Alberto Liptay's CATHOLIC LANGUAGE
}

\begin{abstract}
The purpose of this work is to analyze the catholic language, an artificial a posteriori system devised by the surgeon of the Chilean Navy Alberto Liptay (1859-1922). The project is an exquisite model of rationality, and one of the few systems in Spanish, but it hardly had recognition among its contemporaries, perhaps because the author declared himself only an amateur philologist. A careful study of his numerous contributions offers, however, very suggestive ideological readings of the author that largely determined the configuration of his linguistic proposal.
\end{abstract}

Keywords: Alberto Liptay, naturalist a posteriori constructed languages, catholic tongue, ideology.

Recibido: 29/07/19 Aceptado: 01/10/19

\section{INTRODUCCIÓN: LA PRÁCTICA REVOLUCIONARIA DE LA MEDICINA}

Las referencias biográficas sobre Alberto Liptay (1859-1922), además de fragmentarias, contienen algunos errores acerca de su origen y su trayectoria profesional. En los escasos trabajos en los que aparece mencionado, figura como médico y lingüista chileno ${ }^{2}$; sin embargo, el propio Liptay reconoce su origen extranjero (sin aludir al país de procedencia) al menos en dos ocasiones: la primera, en la conferencia titulada Sovre la posibilidad de un idioma internacional obtenido por sufragio universal, impartida en el I Congreso Científico de Valparaíso Sovre la V i la B en castellano, donde agradece la oportunidad

2 Pei 1958: 126; Salomón 2005; Payàs 2008: 23; Sagredo 2010: 247; Hassler 2017: 118; Alvar 2019. En otras referencias, como en Die International Auxilliary Language Association (http://instituto-erasmo.de/interlingua-plansprache/was-ist-interlingua), figura erróneamente como argentino, lo que sorprende en una asociación dedicada a la Interlingua, que debería ser rigurosa respecto de los autores que trata. 
[...] no solo para acer propaganda por un problema eminentemente umanitario, como lo es sin duda alguna el de un idioma internacional, sino, sovre todo, para poder espresar públicamente mis sentimientos de la mas profunda gratitud acia mi patria adoptiva por mi envío a Europa, i de rendiros cuenta, al mismo tiempo, del resultado qe otuve en este sentido durante mi estadía en aqel continente. (Liptay 1893: 76)

La segunda ocasión, en una pequeña biografía de quince páginas (Moïse ou Malthus? Référendum pro veritate et libertate s.f.) que Liptay redacta como defensa durante su proceso judicial (véase más adelante); a partir de este texto, añadido posteriormente a su polémica obra Pour et contre Malthus (1911: 14-23), puede trazarse su peculiar e interesante biografía.

Aunque no menciona su lugar de nacimiento, los datos sobre su formación en medicina permiten asociar el apellido Liptay (o Liptak) con el barón Karoly Liptay (Liptak) de Pesth (Hungría), con seguridad, su padre, también médico y homeópata. Alberto Liptay estudió medicina y cirugía en Viena (1875?-1877). Probablemente por razones políticas, tuvo que emigrar a los Estados Unidos, donde terminó sus estudios en la Universidad del Estado de Michigan y se doctoró (según figura en la portada de algunas de sus obras) en 1882 con una tesis sobre la figura del homeópata Samuel Hahnemann (Hahnemania: To the greater glory of Sam Hahnemann, the "Messiah in medicine"), amigo personal del barón de Liptay. La vinculación con Chile, que no se interrumpirá nunca, se establece gracias a la amistad con Federico Puga Borne, médico cirujano, profesor y político chileno, quien, dadas las dificultades económicas de Liptay, recomendó su ingreso como cirujano en la Marina de guerra chilena, en plena guerra del Pacífico.

A pesar de carecer de formación filológica específica, Alberto Liptay tenía un conocimiento notable de varias lenguas (latín, griego, húngaro, español, inglés, alemán, francés) y una vasta cultura humanística que le mereció el reconocimiento de algunos lingüistas contemporáneos, como el español Eduardo Benot o los alemanes Max Müller y Rodolfo Lenz -este último afincado en Chile; con estos últimos mantuvo siempre una estrecha relación por sus tendencias ideológicas pangermánicas. Probablemente, la primera redacción de su proyecto de lengua católica se esbozó en Chile bajo la supervisión de Lenz, quien debía encargarse luego de revisar la parte teórica de la edición alemana que apareció en 1891.

Bajo la protección del Presidente de la República de Chile, Domingo Santa María, a quien dedica su obra El darwinismo. ¿Cuál es la posición del hombre en el Universo? (Valparaíso 1888), gozará de ciertas prerrogativas políticas: con el pretexto de perfeccionar sus conocimientos sobre enfermedades venéreas, Liptay fue enviado a París donde, entre 1899 y 1890 , formó parte de la Comisión Naval de Chile, dirigida por el almirante Juan 
José Latorre (O'Ryan 1886: 93), que ocupaba entonces el cargo de ministro de Relaciones Exteriores; en esta ciudad francesa publicó la primera edición de la lengua católica (1890) y las versiones en francés de 1892 y 1897. Tras el cambio de régimen chileno en 1891, Liptay abandona su puesto en la Comisión Naval, aunque seguirá viviendo en París, donde no podrá ejercer la medicina por una cuestión legal relativa a la equivalencia y convalidación de sus títulos académicos.

Fue miembro de la Sociedad Científica de Chile y miembro correspondiente para Chile de la Société des Gens de Lettres, de la Société Philologique y de la Société de la Paix Universelle, pues supo rodearse de personalidades científicas muy relevantes, como Frédéric Passy, fundador de la Liga Internacional de la Paz (1867) y futuro premio Nobel de la Paz (1901), Charles Richet, pacifista liberal, presidente de la Societé française d'eugénique (1912) y premio Nobel de medicina en 1913, y de Edmond Thiaudière, miembro de la Societé des Gens de Lettres. En esta época se dedica fundamentalmente a la divulgación científica y llega a ser un comprometido defensor del neomaltusianismo radical, lo que le ocasionará serios problemas con la justicia francesa. El neomaltusianismo defendía la contracepción en determinados contextos de profilaxis sexual y de higiene; y Liptay, que había conocido las terribles consecuencias de las enfermedades venéreas cuando ejerció como médico de la Armada, lo lleva al extremo de la eugenesis. Entre 1903 y 1908 inicia una serie de publicaciones ${ }^{3}$ tanto para concienciar a médicos y juristas de la necesidad de controlar la prostitución clandestina -que afectaba especialmente a las clases sociales más desfavorecidas-y los embarazos no deseados, como para justificar una profilaxis sexual radical que extendía su acción al control de la procreación en los casos de enfermedades hereditarias:

Aussi proclamons-nous que la prophylaxie sexuelle est tout aussi légitime que l'effort de se préserver de n'importe quelle maladie et nous revendiquons non seulement le droit légal mais encore le devoir moral $\mathrm{d}^{\prime}$ employer tous les moyens dans notre pouvoir non seulement pour

\footnotetext{
3 La lista de sus publicaciones médicas es considerable: Le mystère posthume; causeries médicales sur la mort \& la survie 1903; La préservation sexuelle 1906, obra que conoció más de seis ediciones; Bréviaire de la femme enceinte 1907; Prophylaxia sexualis; causeries médicales sur la préservation et les préservatifs sexuels: d'après les données les plus récentes de la science française et étrangère 1908. Además de sus tratados médicos, escribió sobre darwinismo, pacifismo, novelas humorísticas y una obra pornográfica sobre embrioteratología: La vie sexuelle des monstres: avec mille et une observations curieuses sur leurs organes génitaux 1904. Estas obras están disponibles en la Biblioteca Central de Medicina de París.
} 
empêcher la transmission de toute contagion quelle qu'en fut l'origine, mais encore pour supprimer toutes les souffrances quelle qu'en fut la nature et toutes les misères quelle qu'en fut la source! Aussi ne nous arrêterons-nous pas à mi-chemin dans notre définition de la prophylaxie sexuelle comme la science qui consiste à prévenir les maladies des organes génitaux de l'homme et de la femme, mais, élargissant encore cette définition courante, nous étendrons les maladies, que la prophylaxie sexuelle doit prévenir, des affections transmissibles par la copulation, à toutes les maladies transmises avec plus ou moins de certitude par le père ou la mère et presque fatalement par les deux parents à leurs rejetons. Mais nous trouvons que même cette définition élargie est encore trop étroite, puisqu'elle n'envisage que l'aspect pathologique du problème,

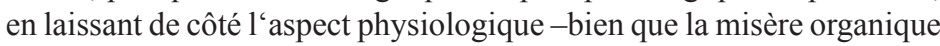
(maladie) et la misère économique (dénuement) s'équivalent au point d'être des états convertibles- et nous concluons à la nécessité logique d'étendre la sphère d'action de la prophylaxie, de la prévention de la maladie, à la prévention de tout mal qui puisse résulter de la fonction génésique. (Liptay 1906: 4)

El neomaltusianismo de Liptay no podría entenderse sin tener en cuenta también el poderoso movimiento feminista de la época, que reivindicaba, además del derecho al voto y a la educación, la libertad para decidir sobre la sexualidad y la maternidad ${ }^{4}$. En Bréviaire de la femme enceinte (1907), bajo la apariencia de recomendaciones sobre embarazos saludables, Liptay oculta, en realidad, argumentos para disuadir a las mujeres de la procreación, considerada por Liptay casi como una patología.

Entre 1911 y 1913, Liptay se enfrentó a varios procesos judiciales, acusado de atentar en sus publicaciones contra la moral pública, pues se entendió que sus argumentos sobrepasaban el ámbito de la contracepción y la profilaxis sexual para encubrir una incitación deliberada a la liberación sexual femenina mediante el autoaborto ${ }^{5}$ las prácticas eugenésicas. El 18 de enero de 1913 fue condenado a tres meses de prisión y a una multa de 200 francos (Hello 2016: 217; véanse algunos extractos del proceso en Gouédart 1993: 200-206). Desde 1912 (muere en 1922) no volverá a publicar nada;

4 La organización feminista mayoritaria, el Conseil national de femmes françaises, fundado en 1901, agrupaba cerca de 80 asociaciones. Entre las integrantes más reconocidas están Marie Huot, la primera en utilizar la expresión Grève des ventres (Ronsin 1980).

5 Para Liptay (1911: 173) "[...] si l'avortement est moralement justifié, ce n'est que comme expédient ultime, comme solution «humanitaire» urgente, et c'est aussi parce que sa fréquence n'est que le symptôme d'une organisation sociale profondément inégalitaire". 
pero esta trayectoria de prácticas médicas libertarias y su comprometida defensa del feminismo tendrán un reflejo en el diseño del género gramatical en la lengua católica, como se verá.

\section{LA LENGUA CATÓLICA EN UN SIGLO DE LENGUAS}

El primer esbozo de la lengua católica-La lengua católica ó sea proyecto de un idioma internacional sin construcción gramatical-se publicó en 1890 en París. Pero este proyecto glotogenético tiene varias versiones que coinciden en lo sustancial y solo difieren en los ejemplos que se utilizan de cada lengua; así, existe una edición en alemán, publicada en 1891: Eine Gemeinsprache der Kulturvölker (Leipzig); y otra en francés, de 1892: Langue catholique. Project d'un idioma international sans construction grammaticale (Paris). En la edición española de 1890, Liptay anuncia una versión en inglés (The future language of civilized humanity) que no he podido localizar; probablemente no se publicó. Posteriormente, en 1893, en las Actas del I Congreso Cientifico de Valparaíso Sovre la Vi la B en castellano, que versó sobre la polémica reforma ortográfica chilena, se incluye una ponencia de Liptay titulada Sovre la posibilidad de un idioma internacional obtenido por sufragio universal. Cuatro años más tarde, en 1897, escribió un opúsculo ${ }^{6}$ de treinta y seis páginas, en edición del propio autor, titulado La possibilité d'un idioma international sans grammaire, que en lo esencial es un resumen de la edición en español planteado en forma de diálogo.

Como se observa en la relación de títulos, el adjetivo católica solo se mantiene en las ediciones española y francesa. El autor aclara en los prólogos respectivos que dicho adjetivo carece de connotaciones religiosas y debe ser entendido en su significado etimológico griego de 'universal'. Liptay suprimió la denominación católica en ediciones posteriores; así, en la versión alemana de 1891 figura lengua común (Die Gemeinsprache der Kulturvölker), e idioma internacional en el opúsculo francés de $1897^{7}$.

\footnotetext{
6 En esta obra anuncia la próxima aparición de un Vocabulaire mnémonique de la langue anglaise y su traducción al castellano (Vocabulario nmemónico de la lengua inglesa) que posiblemente versaran sobre la lengua católica con ejemplos ingleses. No hay rastros de esta publicación más que las referencias que hace el propio Liptay.

7 "Para evitar, pues, críticas a mi proyecto cosmoglótico basadas únicamente en el título, talvez [sic] inadecuado de Lengua Católica, tuve buen cuidado de cambiar este título ambiguo
} 
El proyecto no pasó desapercibido en los foros científicos europeos ${ }^{8}$; a fin de cuentas, la lengua católica es uno de los muchos ejemplos de lenguas construidas que se diseñan en el siglo XIX, el siglo más prolífico en la invención de sistemas lingüísticos a posteriori (Galán 2012). No es fortuito que el auge de estos proyectos a posteriori tenga lugar en plena revolución histórico-comparativa, pues este paradigma favoreció la búsqueda de raíces y procedimientos gramaticales comunes que pudieran anclar el sueño de una lengua internacional. No obstante, frente a la visión romántica de los primeros comparativistas alemanes que interpretaban la evolución lingüística como la degradación de un legado transmitido a la humanidad que había que rescatar, otros lingüistas (Jespersen (1894), por ejemplo), entienden la evolución como un progreso histórico hacia la simplificación, que es el objetivo del proyecto de Liptay.

A partir de las recomendaciones sugeridas por la Société Internationale de Linguistique (1856) y la American Philosophical Society (1887), las nuevas lenguas se atendrán a principios estructurales muy básicos: si son lenguas pensadas para facilitar la comunicación internacional, deberán suprimir las irregularidades y simplificar y unificar sus sistemas gráficos; en consecuencia, se exigirá que la ortografía sea fonética; el alfabeto, latino; la gramática, simple, y el vocabulario, indoeuropeo, preferentemente sustentado en raíces latinas. Y es en la selección del léxico donde las lenguas a posteriori muestran mayores divergencias; así, en el mismo período, conviven tres modelos lingüísticos: en primer lugar, aquellos diseños basados en lenguas muertas, como el latín: lingua et latinesce (Henderson 1888), kosmos (Lauda 1888), novilatin (Beermann 1895), latino sine flexione o

en la edición alemana, edición dedicada, con su beneplácito, al gran savio i célebre profesor Max Müller, qe se dignó presentar i recomendar mi proyecto a sus compatriotas eruditos". (Liptay 1893: 101)

En la revista La ciudad de Dios, y en el mismo año de la publicación de la obra en español (1890), se escribe una elogiosa reseña, si bien se critica que el autor "[...] no ha sabido, al universalizar, desprenderse de la educación española de su oído, pues á un francés y más á un inglés, le sonarán á español muchos de los vocablos católicos" (1890: 607). También ese mismo año La farmacia española se hace eco de la publicación de la obra (1890: 633). Años más tarde, tal vez por la repercusión que tuvo el Congreso de Valparaíso de 1893 sobre la reforma ortográfica, la propuesta de Liptay recibió críticas muy favorables: en La ilustración española y americana (1894: 74) se menciona como "un ingenioso y útil proyecto". En la edición francesa de 1892, Liptay incluye otras críticas elogiosas: entre la prensa francesa destacan los comentarios publicados en Journal des débats (23/07/1890), Le Matin (15/07/1890), Revue des livres nouveaux (15/07/1890), Gil Blas (10/07/1890) o en Revue du monde catholique (octubre 1890), quizá atraída por el nombre de la lengua; no obstante, en la revista Modern Language Notes (1894: 86-91) se publica una dura reseña de Liptay y, por extensión, de todos los proyectos naturalistas de fines de siglo. 
interlingua (Peano 1903); en segundo lugar, los diseños que adoptan lenguas vivas simplificadas ateniéndose a criterios puramente pragmáticos, tales como el poder económico de la nación que la emplea, el peso de su historia o el número de hablantes; de ahí que las lenguas utilizadas sean las lenguas europeas más poderosas, como el francés (langue facile, Henderson 1889), el inglés (word english, Bell 1888), el italiano (lingua franca nuova, Bernard 1888) y el español (lengua española universal, José López Tomás 1918) ${ }^{9}$; por último, se proponen diseños que reúnen el léxico más común y regular de varias lenguas, como el volapük (Schleyer 1879), construido sobre raíces de lenguas naturales deformadas convencionalmente, y el esperanto (Zamenhof 1887), uno de los sistemas a posteriori naturalistas más consolidados.

\section{DE LA CATÓLICA IDEOLOGÍA}

De los tres modelos de construcción de lenguas presentados en el apartado anterior, el que tenía más posibilidades de subsistir era el último, fundamentalmente porque las lenguas romances más internacionales ya compartían en mayor o menor proporción un radicarium latino ${ }^{10} \mathrm{y}$, por tanto, gran parte de los elementos de una lengua artificial así creada existía virtualmente en las lenguas vivas más extendidas. Este es el argumento que utiliza Liptay para defender la viabilidad de la lengua católica, un proyecto -afirma- cuya única originalidad estriba en "la exclusión de toda originalidad": la lengua universal no ha ser inventada, sino descubierta, pues subyace en formas aparentemente disímiles del vocabulario internacional grecolatino:

[...] nos apresuraremos á declarar al lector impaciente que al estampar en la primera página de este libro el lema: La única originalidad de este proyecto es la exclusión absoluta de toda originalidad, partimos del

\footnotetext{
Algunas de las lenguas a posteriori creadas por españoles han sido estudiadas por Calero Vaquera (1996; 2010); Velarde Lombraña (1987; 2000) y Galán (2012; 2016).

10 Jacob Grimm, por ejemplo, redactó poco antes de su muerte un Esbozo de lengua universal (1860) en el que sugería utilizar el latín como hilo conductor para la formación de las raíces esenciales. El texto íntegro, traducido al francés, se recoge en Couturat \& Léau (1903: 121-127).
} 
principio que, aunque no hay todavía una lengua universal, existen sin embargo y abundan voces universalmente empleadas. (Liptay 1890: 133)

En la lengua católica está en la humanidad entera; ella no es una ficción, sino un hecho; se halla repartida entre los idiomas cultos. No es más que un castellano, un portugués, un italiano, un francés, un rumano y aun un inglés o alemán simplificado, o mejor dicho, un término medio de todos ellos y por consiguiente fácilmente comprensible por todos aquellos cientos de millones que poseen cualquiera de estas lenguas [...] nuestra creación está no solo en nuestro cerebro sino en el de la humanidad entera, formando parte integrante de su civilización! Esta lengua católica no es una ficción sino un hecho y no por encontrarse repartida hoy día entre todas [sic] los idiomas cultos es menos evidente su existencia; justamente á causa de esta difusión de sus elementos es porque se escapó hasta ahora á nuestra vista y porque no puede considerarse todavía como una cosa concreta y tangible, como un idioma especial con sus diccionarios, gramáticas y demás accesorios propios [...]. (Liptay 1890: 241)

Esta lengua tiene la ventaja - dice Liptay (1890: 5) - de ser un proyecto filantrópico en el que debería implicarse la comunidad filológica internacional y “[...] todas las personas ilustradas y aficionadas á las letras que quisieran cooperar á la realización del humanitario fin que persiguimos [sic]" (Liptay 1890: 243) para no depender de la arbitrariedad de un único inventor. Ahora bien, este pretendido altruismo que hace descansar la supervisión de la lengua en una tarea colaborativa muestra también la faceta más oscura del autor: la del intelectual no reconocido por la academia ${ }^{11}$ que es consciente de que el amparo económico de sus mecenas chilenos y franceses es efímero.

De ahí la captatio que cierra el prólogo de la edición de 1890 en el que declara no aspirar ni a gloria ni a reconocimientos, pues tan solo es un "aficionado á las letras". Sin embargo, utiliza un juego sutil entre el plural de modestia y la tercera persona para aludir a sí mismo:

[...] si aceptamos como racionales las ideas de este hombre y como meritorias sus aspiraciones; si las encontramos, después de un examen imparcial, no solo practicables sino aun de fácil ejecución; si nos convencemos de la utilidad general de su obra y de las inmensas ventajas que redundarían de ella en pro del bien público; entonces no bastan nuestros aplausos para la realización de su proyecto filantrópico, por lisonjeros que sean ellos para el amor propio del iniciador. No; lo que

11 "Je suis, malheuresement, encore le seul le représentant en France de ces idées ou plutót de la maniére objective de les réaliser" (Liptay 1897: 31). 
se nos exige entonces es nuestra cooperación más ardua y entusiasta que debemos ofrecer espontáneamente para la realización pronta y radical y no solo paulatina y parcial, de un proyecto tan vasto que abarca la humanidad entera por lo elevado de las miras y la universalidad de los beneficios, materiales y morales, á que aspira. En una obra de esta naturaleza, es decir de una índole tan altruista, estamos llamados á contribuir todos según nuestros esfuerzos [...] á la creación de un monumento tan gigantesco como lo sería una lengua universal destinada á marcar una nueva era de confraternidad entre los pueblos civilizados del globo terrestre. (Liptay 1890: 2-3)

Realmente Liptay solo recibió un apoyo -tibio- del lingüista y orientalista alemán Max Müller, a quien había encargado la redacción del prefacio para la traducción alemana de su proyecto que debería hacer Lenz. En la edición francesa de la lengua católica (1892), Liptay incluye una carta de Müller en la que este, si bien reconoce que la propuesta de Liptay tiene cierto interés, duda de la viabilidad de una lengua así planteada:

Votre idée de choisir des mots radicaux presque universellement compris par les gens instruits est excelent, et l'articulation grammaticale que vous proposez est très praticable, quoiqu'on puisse proposer çà et là quelque chose de plus simple et de plus pratique [...] je crois qu'une langue catholique comme la vôtre sera très utile pour la communication mais prendra-t-elle jamais la place d'une vraie langue vivante? J'en doute, en considérant ce qu'est la nature humaine. Cependant, s'il est possible d'assembler des millions de têtes sous un même bonnet, je crois que votre proposition à la meilleure chance de succès. (Liptay 1892: 5)

En términos semejantes se expresa Benot, quien, no sin ironía, escribe:

Nada más racional que el proyecto de LIPTAY; nada más fácil relativamente [la cursiva es de Benot]. Pero el problema está aún por resolver, porque el lenguaje universal no ha sido inventado todavía. Y, caso de serlo en día afortunado, ¡cuánto tiempo no exigiría el llegar á un gran convenio internacional en cuya virtud se generalizara! ¿Qué gobierno, por autoritario que fuese, se atrevería á declararlo obligatorio, ignorando si al fin sería aceptado por todas las naciones? Y ¿no lo rechazarían constantemente los pueblos incapaces de pronunciar ciertos sonidos que nos son muy fáciles y para quienes resultan refractarias nuestras construcciones? En Filipinas nos presentan los chinos allí establecidos desde hace muchos años, palpables ejemplos de su incapacidad increíble para pronunciar nuestros sonidos y hablar según nuestra sintáxis. Benot (1892: 259) 
Su gran decepción fue la oposición radical del filólogo Gaston Paris, que llega a proponer adoptar una especie de volapük -lengua artificial que Liptay despreciaba profundamente- antes que la lengua simplificada de Liptay (Liptay 1897: 31).

Esto explica sus amargas críticas contra los filólogos de cátedra, "enemigos pasivos de cualquiera causa humanitaria" (Liptay 1890: 242) frente a los filólogos de profesión, "consejeros de la asociación cosmoglótica" (Liptay 1897: 29), que apoyan proyectos semejantes al suyo y reconocen la labor de sus creadores en otros países. Aunque Liptay afirma que solo busca el reconocimiento moral, en el fondo lamenta la escasa financiación de que dispone y solicita, de manera directa o indirecta, colaboración económica:

Notre système a été conçu sans l'aide de personne; loin de considérer une pareille indépendance comme un avantage nous la dénonçons justement comme cause nécessaire de tous les défauts qui abondent dans notre project [...] J'ai, en effect, dépensé une dizaine de mille francs pour ces diverses publications de propagande. (Liptay 1897: 32-33)

[...] il ne faut pas que cette question reste dans son état embryonnaire de projet plus o moins défectueux et ... platonique; il faut, au contraire, que le noyau pousse (crecer) et devienne un arbre et que cet arbre prenne racine d'abord dans le généreux sol français et ensuite dans toutes les terres latines et germaniques, puis finalemente sur toute la superficie terrestre appartenant à la civilisation et à la fraternité. (Liptay 1897: 36)

Las dedicatorias de sus publicaciones a personajes ilustres no surtieron el efecto deseado, si bien están cuidadosamente seleccionados y reflejan tanto la trayectoria intelectual del autor como sus débitos intelectuales. A modo de ejemplo: la primera edición de 1890 (con la fecha de 21 de julio anotada a mano por el autor) se abre con una dedicatoria "Á Su Magestad la Reina Regente de España" y se cierra con otra "A la Memoria de Domingo Santa María", Presidente de Chile entre 1881 y 1886. El opúsculo de 1897 se dedica a "Son excellence l'Amiral don J. J. Latorre", senador de la República de Chile, bajo cuyo mando ejerció como cirujano a bordo del buque Angamos en la Guerra del Pacífico ${ }^{12}$. La edición de las intervenciones en el Congreso de Valparaíso (1893) está dedicada a Diego Barros Arana, autor de la monumental Historia General de Chile en 16 volúmenes (1884-1902), muy comprometido con la reforma educativa chilena.

12 “[...] l'Amiral Latorre a bien voulu s'intéresser à mon projet cosmoglottique et me protéger hiérarchiquement dans mes travaux de propagande”. (Liptay 1897: 32) 
Por otra parte, como ocurrirá en casi todas las lenguas a posteriori del XIX sustentadas en un radicarium latino, la búsqueda de la internacionalidad fue derivando hacia una interoccidentalidad o interlatinidad cada vez más sesgada ideológicamente. Este giro es especialmente llamativo en el análisis que hace Liptay de los proyectos artificiales de los siglos precedentes (1890: 20-31) a los que considera ingeniosos, pero impracticables por su excesiva arbitrariedad y apriorismo.

Estos defectos se acentúan especialmente en el caso del volapük de Schleyer; pese a reconocer -a veces con cierto rencor-su éxito, Liptay condena que el autor sacrificara la racionalidad del diseño en favor de una pretendida universalidad, lo que devino en un "producto inarmónico [...] sin referencia sistemática al fondo común de la elocución universal" (Liptay 1890: 37; Benot se expresa en los mismos términos dos años más tarde (1892: 258)). Solo la lengua católica, en su condición de lengua descubierta tras su devenir histórico, puede aspirar a satisfacer el anhelo de la elocución universal, reflejo, a su vez, del progreso al que aspiran las naciones civilizadas; por ello, aunque su proyecto se pretenda etimológicamente católico, solo será occidental (romance):

L'unité est décidément plus grande parmi les langues romanes que parmi les langues germaniques et c'est seulement un panslaviste ou un chinois qui pourrait s'enthousiasmer pour une langue universelle qui ne soit basée ni sur l'un ni sur l'autre de ces groupes. (Liptay1892: 85)

De ahí que Liptay excluya a todos aquellos pueblos a los que atribuye un estadio inferior de evolución y civilización:

[...] La civilisation [...] ne s'étend non plus sur toute l'humanité, mais à une fraction de celle-ci $[\ldots]$ c'est que cela ne peut être autrement pendant que l'espèce humanine se divise encore en peuples civiliés et en peuples sauvages, et pendant qu'il existe d'un côté des Français et des Espagnols, des Allemands et des Anglais, et d'autres des Nègres et des Cafres, des Bushmen et des Papuas [...] Mais que nous importe que les avantages d'une langue internationale soient restreintes à la moitié de l'espéce humanine, si c'est juste cette moitié qui a élevé l'art et la science, le commerce et l'industrie à une hauteur et à une perfection que l'autre moitié ne soupçonne même pas. (Liptay1892: 85)

Y es el pueblo chino el que recibe las críticas más ácidas e injustas. Sorprenden los juicios de valor que plagan los textos de Liptay cuando se refiere a esta lengua con afirmaciones que rompen su habitual tono conciliador para 
caer en un dogmatismo apodíctico e ideológicamente sesgado ${ }^{13}$. La razón no es otra que el desprecio que le merece el volapük, cuya simplificación fonética, según Liptay, no obedece a su proyección internacional, sino a la incomprensible necesidad de hacerla accesible a otros pueblos (como el pueblo chino) que tienen dificultad para articular determinados sonidos:

Por consiguiente ridículo es y absurdo exigirnos que nos mortifiquemos aprendiendo un idioma chinesco como el volapük, nada más que para ponernos al alcance de los chinos, pueblo tan exclusivista y ultraconservador que aun no ha experimentado la necesidad de un vehículo común para entenderse entre sí, sin tener que recurrir al diccionario y á la escritura. (Liptay 1890: 190)

Pero ni siqiera los nombres de los continentes respetó el vándalo de Schleyer; al contrario, los mutiló sin misericordia; así se trasformaron bajo su pluma sacrílega Europa en yulop, Asia en silop, África en fikop, etc. Naturalmente, nadie save lo qe significa yulop, con escepcion talvez de los qe ablan el llamado pidgin-english, es decir, el ingles ablado no por los pichones, sino por los chinos; en cambio, todo el mundo conoce el nombre de Europa, por ser éste empleado por todas las naciones civilizadas del globo terrestre, i ser, por consigiente, Europa un vocablo universal, de echo i sin decreto especial. (Liptay 1893: 81)

Estas críticas coinciden con las que años más tarde formularán los neogramáticos Brugmann y Leskien en una breve publicación titulada Zur Kritik der künstlichen Weltsprachen (1907), redactada por encargo de la Königliche Sächsische Gesellschaft der Wissenschaften de Leipzig. Brugmann y Leskien niegan que lenguas como el volapük o el esperanto sean realmente fáciles de aprender, pues su fonética y sus patrones acentuales y prosódicos son extraños para los occidentales; por otra parte, aun pretendiendo ser internacionales, el vocabulario y la morfología (por ejemplo, en el caso del esperanto, aunque esta crítica podría hacerse extensible a la Lengua

13 "La lengua china, por lo visto, es impersonal y concisa al extremo de llegar á ser lacónica y aforística; para expresar que algo no tiene sentido común, el chino dice: "(Esto) no constituir (un) término", ó "(la) frase no (es) completa". En lugar de tomar aliento y decir de un tirón: Hace muchísimo tiempo, Señor, que he tenido que renunciar al infinito placer de verlo en esta su humilde casa; el chino ganguea simplemente: Kiéu-uei = mucho tiempo volver espalda" (Liptay 1890: 66). Sorprende este juicio tan escasamente científico cuando la lengua china ha sido el modelo por excelencia para las construcciones filosóficas de épocas precedentes. 
Católica) son muy similares a los de las lenguas romances; por último, si una lengua artificial se crea para facilitar el intercambio internacional, este mismo espíritu es el principio de la desaparición de la lengua: si está en manos de los hablantes, es inevitable que estos la modifiquen (como ocurrió con el esperanto); por tanto, una lengua artificial solo lo será mientras no se ponga en circulación:

Die Sprache ist, wie alles kulturelle Tun, ununterbrochen der Entwicklung, dem Wandel unterworfen. Dabei sind in jeder Sprachart, wie sie über ein irgendwiegroßes Terrain, eine irgendwiegroße Verkehrsgenossenschaft verbreitet ist, stets sowohl zentrifugale, differenzierende Kräfte wirksam als auch zentripetale, ausgleichende [...] Die Ergebnisse der Wirksamkeit dieser beiden einander entgegengesetzten Tendenzen machen bei jeder Sprachart, die wir als eine Einheit anzuschauen pflegen, einen Hauptteil ihrer Geschichte aus [...] Hierbei ist nun für uns von Wichtigkeit, daß die Fremdsprache nie rein bleibt, namentlich nicht im Lautlichen, in Wort- und Satzfügung und im Phraseologischen. (Brugmann y Leskien 1907: 23-24)

\section{LA LENGUA CATÓLICA COMO DESCUBRIMIENTO}

Liptay argumenta que el lenguaje es un producto único de la razón humana que se ha desplegado en formas disímiles desde un mismo origen. El objetivo del lingüista, y de todo constructor de lenguas con proyección internacional, por tanto, es aplicar un método racional para descubrir precisamente la base común y natural de las lenguas, lo que supone un rechazo radical a la glotogénesis arbitraria de sistemas artificiales como las lenguas filosóficas o el volapük. Su naturalismo occidental tomará como punto de partida el latín, cuyas raíces pueden rastrearse en todas las lenguas europeas.

Pero este latín no puede ser el dialecto clásico que se obliga a estudiar en los colegios "disecando y analizándose su cadáver artificialmente conservado", sino los dialectos modernos "uno de los cuales constituye precisamente nuestra lengua materna" (Liptay 1890: 72):

[...] i [lo] qe ablamos todos nosotros es el latin, tal cual se a modificado en el trascurso de los siglos para satisfacer las exigencias del progreso umano. Donde todo adelanta, la Lengua sola no pudo averse qedado atrás, i en su evolución adaptóse inconscientemente a las condiciones orográficas i climatolójicas de cada pais i a las necesidades e idiosincrasias de cada pueblo. (Liptay 1897: 90) 
Y, en efecto, el trabajo de Liptay será compilar elementos lingüísticos ya existentes, universalmente reconocidos y empleados en las lenguas. De ahí el lema que aparece en todas sus publicaciones y su rechazo a la invención arbitraria:

En imprimant sur la première page de tous mes travaux cosmoglottiques la devise que 'l'unique originalité de mon Project est l'exclusion de toute originalité', je suis parti du principe que, quoiqu'il n'y ait pas encore de langue universelle, il y a cependant abondance de mots universellement employés, et qu'il suffirait de compiler ceux-ci afin d'obtenir celle-là. (Liptay 1897: 13-14)

El principio de racionalización que impera en la lengua católica es similar al de otros proyectos naturalistas más elaborados, como el universalglot (1868) de Pirro o el mundolingue (1888-1892) de Lott, proyectos que Liptay no cita $^{14}$, aunque conoce sobradamente; por ejemplo, el mundolingüe es una lengua naturalista que utiliza las raíces latinas más comunes al alemán, inglés y francés, aproximándolas al italiano por su fácil pronunciación. Desde luego, carece de las fatuas pretensiones de universalidad de la lengua católica, pues está pensada para las naciones de Europa Occidental. El vocabulario prima sobre otras cuestiones lingüísticas; así, una vez constituido, podrá fijarse el alfabeto, la pronunciación y la gramática, pues deberá buscarse la uniformidad gráfica antes que la fonética, ya que la ortografía de las palabras es más internacional que su pronunciación y esta lengua está destinada a ser escrita más que a ser hablada. El proyecto es un ensayo provisional, no una lengua cerrada; por este motivo, Lott fundó la Sociedad Internacional para la Lengua Universal con el propósito de que todos los sabios pudieran contribuir en su diseño, lo que recuerda las propuestas colaborativas de Liptay respecto de su lengua ${ }^{15}$.

14 Liptay se queja de haber sido acusado de plagio: “Ainsi Mr. Jules Lott, l'auteur de Lingua International dont la connaissance opportune nous aurait dispensé de proposer une langue catholique, tellement les deux projets se rapprochent, a formulé l'opinion suivante sur le nôtre: «la proposition du Dr Liptay concernat la prononciation de l'alphabet et de l'ortographe, comme aussi en ce qui concerbe le vocabulaire est le meilleur de tout ce qui a été présenté jusqu'ici; sa manière de traiter le verbe me rappelle cependant le volapük»". Conocida la animadversión que sentía Liptay hacia el proyecto de Schleyer, es probable que la cercanía que advierte Lott entre el sistema verbal del volapük y el de Liptay irritara a este último y, en consecuencia, decidiera no aludir a los trabajos del austríaco sobre el léxico internacional.

15 Tampoco cita Liptay la lengua universal de E. Heintzeler (1890-1893) con la que las coincidencias son aún mayores: Heintzeler, como Liptay, defiende que la lengua artificial universal existe en potencia y tan solo hay que activarla utilizando palabras y raíces comunes de las lenguas romances; para esta ingente labor reclama también una comisión internacional 


\subsection{DE LA GRAMÁTICA CATÓLICA}

La gramática de la lengua católica no es especialmente novedosa ${ }^{16} \mathrm{y}$ coincide en lo esencial con la mayor parte de las lenguas naturalistas. Por ejemplo, rechaza las marcas de caso (Liptay 1890: 193), porque este puede expresarse bien mediante preposiciones (de y a para el genitivo y el dativo, respectivamente), bien mediante la posición (el acusativo ocupa una posición postverbal en la frase). Mantiene las formas del artículo determinado (lo (masc.), la (fem.) y le (si no hay marca de género)) e indeterminado (uno, una, un) y un sistema pronominal que recuerda básicamente al latín: $e o, t u$, ele (con las formas elo (masc.) y ela (fem.) para el singular), y nos, vos, eles (elos (masc.) y elas (fem.)) para el plural. Los pronombres interrogativos y relativos se reducen a las formas invariables qe y qi. También son invariables los adjetivos y se sustantivan añadiendo a la raíz una $-o$ para el masculino, una - $a$ para el femenino, o una $-e$, si no se especifica el género: bono o lo bon ('el bueno'); bona o la bon ('la buena'); bone o le bon ('lo bueno'). Los numerales (Liptay 1890: 203-5) derivan del latín: un, $d v e^{17}$, tre (por ser de pronunciación más común y fácil que tres), quator (por ser más clásica, si bien no más sencilla que otras soluciones, como quat o qatr); quin (pronunciado / kvin/), six (por evitar la forma sex, reservada para sexo), sept(e) (suprimiendo la terminación latina de septem), oct(e) (eliminando la

de intelectuales. Igualmente, defiende que la gramática internacional ya existe o, al menos un acuerdo razonable acerca de qué debe y qué no debe aparecer en ella; por ejemplo, la simplificación (o supresión) de los artículos, el género natural, la declinación única y analítica, la conjugación simplificada, etc.

${ }_{16}$ En Galán (2016: 74-78) hay una descripción más detallada. Es llamativo que Liptay se despache la gramática, que no es más que un esbozo de morfología, en un capítulo heterogéneo titulado Miscelánea (1890: 191-236) en el que hay conceptos gramaticales simplemente apuntados y elecciones muy discutibles. Por ejemplo, en relación con la conservación de las formas del artículo, dice: "[...] la creación del artículo tiene su razón de ser, ya que sirve á especificar el sustantivo, á individualizarlo, á ... qué sé yo de todo lo demás! El lector sabrá mejor que yo, y si no lo sabe, lo ignoramos los dos, mas, no por tal ignorancia deja de ser un hecho la existencia del artículo en casi todas las lenguas cultas" (Liptay 1890: 194); A veces da la impresión de que Liptay calibra si merece la pena el esfuerzo titánico que le supone construir la lengua, pero si alcanzara mayor consideración: "[...] con mucho gusto dedicaría lo que queda de vida a elaborar el vocabulario y completar la gramática de este idioma". (Liptay 1890: 240).

17 Liptay reserva las formas $d v o$ y $d v a$ para marcar masculino y femenino, respectivamente: dve individ ('dos individuos', genérico), dvo senilo ('dos abuelos'), dva senila ('dos abuelas'). Es interesante señalar que el sustantivo al que determina el numeral $d v e$ nunca lleva marca de plural, pues la idea de pluralidad ya está expresada en el numeral. 
- o para evitar la confusión con el morfema masculino); nov (o nove, como el radical sánscrito y latino) y dece.

Los verbos católicos (Liptay 1890: 207-223) pueden conjugarse según dos procedimientos: bien flexionando el verbo con un morfema de persona $(-o,-a,-e$, para el presente de indicativo, por ejemplo) y de número ( $-o s$, $-a s,-e s)$, con lo cual el pronombre no es necesario, bien manteniendo una forma invariable para el verbo, e indicando en el pronombre la persona y el número, procedimiento que recomienda el autor por ser más racional. En este último caso, el verbo (simplificado en una raíz) se sirve de desinencias vocálicas (elegidas por analogía con el latín, el francés y el italiano) para indicar los tiempos, independientemente de la persona y del número, como en esperanto:

$\begin{array}{ll}\text { Presente } & a m-\boldsymbol{e} \\ \text { Pretérito perfecto } & \text { am-á } \\ \text { Futuro } & a m-\mathbf{o} \\ \text { Pretérito imperfecto } & a m-\mathbf{i} \\ \text { Pretérito pluscuamperfecto } & a m-\boldsymbol{u} \\ \text { Futuro perfecto } & a m-\mathbf{a}-\mathbf{o}\end{array}$

La pasiva se construye con el verbo ser (auxiliar invariable y simplificado como -é): eo am ('yo amo') / eo amé ('soy amado'); no obstante, estas formas aglutinadas pueden desarrollarse analíticamente (el é am), procedimiento más adecuado en las formas del pasado para evitar escribir (y pronunciar) dos vocales acentuadas: nos é amá ('nosotros hemos sido amados'), en lugar nos améá. Las formas del subjuntivo y del condicional no difieren del indicativo y se marcan mediante la anteposición de las conjunciones qe o si: qe eo am ('que yo ame'); qe eo amá ('que yo haya amado').

Las mismas formas verbales, sin pronombre, sirven de infinitivo y de imperativo; en este último caso, el pronombre se pospone al verbo: ame tu!, ame el! Para las formas de gerundio, sugiere la terminación-anté o-enté: amanté ('amando'); vendenté ('vendiendo').

Finalmente, admite dos verbos irregulares auxiliares, ser y haber, reducidos a una mínima raíz vocálica (é y $a$, respectivamente) presente en todas las lenguas europeas, cuya conjugación por tiempos es la siguiente:

Verbo auxiliar
Presente
Imperfecto
Perfecto
Pluscuamperfecto
Futuro
Futuro perfecto

$\begin{array}{ll}\text { Ser (é) } & \text { Haber }(a) \\ e ́ & a \\ i & u \\ e i & a u \\ i i & \text { uu } \\ \text { éó } & a o \\ i o ́ & \text { uó }\end{array}$




\subsection{Del alfabeto Católico}

En líneas generales, Liptay sigue las recomendaciones de la Société Internationale de Linguistique (1856) respecto a la pronunciación de los sistemas inventados con pretensiones de internacionalización: el alfabeto es latino, para evitar caracteres extraños a las lenguas europeas, como las consonantes con acento circunflejo del esperanto o las vocales inflexionadas del alemán y del francés (ä, ö, ü). La ortografía es fonética y el respeto a los principios fonéticos le lleva a adoptar algunas decisiones controvertidas, la supresión de la $h$, por ejemplo, como ya habían sugerido Bello y los neógrafos reformistas chilenos respecto a las letras mudas ${ }^{18}$ :

Repetimos que hallamos muy racional la supresión de la $h$, mas, no nos creemos facultado [sic] para suprimirla de hecho ni siquiera en el proyecto internacional que proponemos - ¿con qué derecho? Dejamos por consiguiente á la opción del público la eliminación de la $h$, confiando que desaparecerá de la ortografía como desapareció de la ortología, es decir, que la h será suprimida en la escritura como ha sido suprimida ya en el habla de los pueblos latinos. La conservación provisional en nestro [sic] alfabeto internacional del símbolo de la aspiración gutural en cuestión es una concesión que hace el espíritu progresista al espíritu conservador! (Liptay 1890: 116)

También discute el empleo de la $b$ no justificado ni por la ortología ni por la etimología (como el caso de abogado < lat. advocatus), así como el uso de la $v$ cuando etimológicamente existía una $b$ (maravilla $<$ lat. marabilia). $\mathrm{Su}$ propuesta es que se sustituya, también en la escritura, la $b$ por la $v$ : “[...] escribamos pues saver, recivir, caveza... tal cual se pronuncia y pronunciemos beso, boca, bigote... tal cual se escribe -ya que el sentido común así lo exige! (Liptay 1890: 106-107). Sin embargo, el autor no corrige algunas irregularidades de las lenguas romances que se habían descartado en otros sistemas, como las variaciones entre $c$ y $s$ o entre $g$ y $j$. (Liptay 1890: 110)

Liptay mantiene objeciones semejantes respecto al grupo $c h$, que no es un sonido primitivo, sino una combinación. En su lugar, sugiere el signo

18 En cierto sentido, su modelo naturalista participó indirectamente de la polémica reforma ortográfica de otros compatriotas, como Carlos Cabezón, quien, en 1892, propuso la creación de una ortografía rrazional basada en la "[...] kabal korrespondencia entre los sonidos elementales de la lengua, i los signos o letras ke an de rrepresentarlos" (Bello 1884: 387). Véase también Cáceres y Rojas (2019). 
internacional $\int$, correspondiente a la sh inglesa o a la sch alemana; no obstante, como su propuesta es personal, recomienda utilizar la letra rusa ya existente $-\mathrm{h}-$ para el fonema / $/$ /. En las voces internacionales helénicas que tuvieran ese sonido se suprimiría simplemente la $h$ (caos, caracter) hasta que esa $c$ se reemplazara por una $k$, más racional (Liptay 1890: 112). La misma reducción se aplica a otros grupos consonánticos dobles, como $p h(>$ $p)$ o $l l(>l)$. Para justificar esta última simplificación, argumenta que, como es un sonido nacional, no puede formar parte del alfabeto católico (al igual que la ñ, que representa como ny); además, en castellano existen dobletes que podrían emplearse en casos de ambigüedad, como llamar/clamar, lleno/ pleno, llano/plano, etc. (Liptay 1890: 119)

Con el mismo criterio, la $w$ queda también excluida del alfabeto católico porque es una letra propia del inglés y del alemán y, por tanto, su uso se limita a las voces prestadas que la contienen (Liptay 1890: 127). Respecto a las velares, la $g$ tendrá siempre una pronunciación suave, aun delante de $e / i$; así, se escribirá sige, ginda, suprimiendo la vocal $u$, porque no se pronuncia; por su parte, el sonido fuerte se representará mediante una $j$ (como proponía, por ejemplo, Bello). Por último, determina prescindir de la $z$ porque es una letra “tan heterófona como lo es el símbolo en cuestión". (Liptay 1890: 129)

\subsection{Del vocabulario católico}

El vocabulario de la lengua católica no está terminado, si bien Liptay deja claro el método que ha de seguirse para su elaboración: como es una lengua naturalista, selecciona las raíces y sufijos que considera realmente internacionales (hay que entender occidentales de procedencia latina) porque existen en las lenguas europeas más habladas bajo formas diferentes que hay que unificar. Este es el sentido del descubrimiento que impregna su lengua frente a la invención arbitraria y gratuita de otros diseñadores artificiales.

Entre los sufijos más productivos, cuyo uso permitiría construir unos 2000 vocablos católicos, Liptay selecciona -al, -or (con variantes -sor y -tor) y -on (con variantes - cion y -sion) (Liptay 1890: 136-140). En algunos ejemplos del francés y del inglés se aplica una modificación gráfica, según el criterio de la ortografía fonética, aunque no siempre es sistemático (Galán 2016: 69-71). La lista se incrementa con otros sufijos que pueden haber sufrido alguna modificación en las lenguas europeas, como -ant (e); -ent $(e, o, a)$; -ible y -able; -ment $(e) ;-u t(o)$ e -it $(o) ;-u r(a)$; -in $(a)$; ism $(o) ;-i c(a, o) ;-d a d$ (-tat en alemán, -ta en italiano, -té francés y -ty en inglés);-ancia, -enciu (-ance, -ence, -anz, -enz). A la suma de voces católicas ya consignadas podrían añadirse vocablos de empleo universal, 
o "voces universales por excelencia ó sea... arquicatólicas!" (Liptay 1890: 149), como alkol, kafé, chocolat, cigar, tabak, kiosk, kán (Kahn), pasha, sultan, siest, sport, club, jockey; y algunos topónimos e hidrónimos, como Egipt, France, París, Vien, Berlin, London, Danub o Rin. En estas palabras internacionales, Liptay no aplica siempre la ortografía fonética (escribe alkol, kafé, pero chocolat o club; jockey y no jokey, pues en el grupo -ck-no se pronuncian las dos letras); además, la forma en que transcribe las palabras a veces no se corresponde con la de la lengua en que se usan (y desde la que se han exportado como internacionales), por lo que en la universalidad se desliza siempre alguna lengua interpuesta; tal es el caso, por ejemplo, de París (castellanizada con tilde), frente a Berlin, France o London.

\subsection{Del GÉNERo Católico}

Para Liptay existe una correlación natural entre sexo biológico y género gramatical, correspondencia que tiene un reflejo directo en las terminaciones de las palabras:

[los sustantivos tendrán el género] natural que corresponde al sexo de los seres animados y será siempre neutro cuando no signifique hembras ni varones y aun estos serán considerados como neutros cuando no nos preocuparemos [sic] especialmente del sexo al cual pertenecen, como generalmente en el caso de los animales y aun á veces en el de los seres humanos como en los niños, por ejemplo, pudiendo ser una guagua aun en castellano indistintamente hombre ó hembra. (Liptay 1890: 154)

Liptay distingue tres géneros: neutro o genérico (sin terminación), masculino (terminado en $-o$ ) y femenino (terminado en $-a$ ). Escribo conscientemente terminación y no desinencia puesto que, para evitar una equiparación errónea de género y sexo cuando no corresponde, Liptay suprime estas vocales finales en todos los nombres que designan entidades inanimadas. Así, en la lengua católica se escribirá vin, lun, terr, mund o univers para designar respectivamente vino, luna, tierra, mundo o universo (Liptay 1890: 175), puesto que carecen de sexo. Y distinguirá "por ridículo que parezca al principio" poeto, perso, naturalisto, profesoro como formas masculinas de los vocablos femeninos poeta, persa, naturalista y profesora, cuyos genéricos son poet, pers, naturalist y profesor, respectivamente: "La frase «Se necesita un buen profesor» significará exclusivamente que se requiere una persona competente dedicada a la enseñanza, sea esta hombre o mujer". (Liptay 1890: 176) 
Siendo consecuente con esta distribución vocálica, suprimirá las terminaciones $-a$ y $-o$ de todos los sufijos en aquellas palabras que no designen entidades animadas, si bien no siempre las soluciones son racionales ${ }^{19}$; por tanto, escribirá argument, moment, atribut, tonsur, asignatur, motiv, divin, etc. porque "Un argumento no tiene sexo y un momento, si es posible, menos todavía". (Liptay 1890: 141-2)

La distinción de géneros se aplica rigurosamente en el ámbito del parentesco, pero se lleva a extremos delirantes en los vocablos que designan algunas profesiones y objetos de uso cotidiano, palabras en las que la base lingüística común es menos perceptible, pues su empleo frecuente ha provocado que las modificaciones que han sufrido en cada lengua sean menos uniformes.

Para organizar el campo del parentesco, Liptay parte de la raíz latina hom (término genérico que designa 'ser humano') ${ }^{20} \mathrm{y}$ diferencia entre homo y homa (plural homes), vocablos también genéricos que solo incluyen la especificación del género, sin aludir a la edad o al estado, como ocurre, por ejemplo, en los pares señora / señorita (español), Frau /Fräulein (alemán); Mrs. / Miss (inglés); madame / mademoiselle (francés); senhora / senhorita (portugués) o signora / signorina (italiano), cuyo uso se establece en relación con el estado civil. El rasgo semántico 'edad', más los atributos naturales que le corresponden, se plasma en tres oposiciones: viro(s) $(<$ lat. vir $)$, 'hombre robusto, vigoroso, adulto', frente a vira(s), 'mujer adulta, valiente o fecunda' (Liptay 1890: 161; nótese que la fecundidad solo se atribuye a la mujer); infanto(s) / infanta(s) para designar, respectivamente, 'niño(s)' / 'niña(s)' (infante(s) sería el genérico); y senilo(s) / senila(s), para aludir a 'anciano(s)'/'anciana(s)'. Estos ejemplos también ilustran la formación regular del plural: se añade $-s$, si la palabra termina en vocal (infantos, infantas), $\mathrm{y}-e s$, si termina en consonante (homes).

19 Las soluciones católicas para regular los sufijos son las siguientes (Liptay 1890: 143): $-u t o,-i t o>-u t$, -it (atribut, absolut, tribut, institut);-ivo >-iv (preventiv, motiv, sustantiv, primitiv); -ismo >-ism (silogism, aforism); -ura, -ina > -ur, -in (natur, literatur; carabin, doctrin); -ulo> -ul (cálcul / calcul; no señala cómo debería resolverse el acento); en otros sufijos, sin embargo, no suprime la supuesta terminación de género y decide adaptar formas inglesas o francesas: -orio $>-r y$ (directory); -ario/-aria >ár (diccionár, inventár, breviár, seminár);-cio>is (servís, edifís, solstís), lo que resulta una inconsistencia, pues en la lengua católica la $-\mathrm{s}$ es siempre marca de plural. Liptay no lo resuelve y, aunque reconoce esta contradicción, añade: "no por ser reservada para la designación del plural en la desinencia dejará de prestarnos importantes servicios como parte integral ó radical del vocablo en el singular". (Liptay 1890: 145)

20 En Liptay (1890: 156-159) se incluye una interesante disquisición sobre la etimología de 'hombre' en varias lenguas europeas. 
La coherencia de su propuesta se mantiene en todos los términos del parentesco: entre las formas católicas parento ('padre') y parenta ('madre'), fila ('hija') y filina ('nieta'), existe una conexión formal más transparente y racional que entre padre y madre o hija y nieta. La misma relación transparente hay entre maritar (verbo) y marito / marita (sustantivos), más acordes estos últimos que la diferencia, por ejemplo, entre marido y mujer o que la solución arbitraria del esperanto: patroj ('padres'; neutro) /patrico ('padre'; masculino) /patrino ('madre'; femenino).

La relación familiar se amplía a hermano, distinguiendo entre frat ('hermano' genérico) / frato(s) (masculino) y frata (s) (femenino); para los primos (por su relación próxima), mantiene la misma base y añade el prefijo con-, que ha de entenderse en el sentido de 'casi', pues un primo es casi un hermano: confrate (genérico) / confrato (masculino) / confrata (femenino). (Liptay 1890: 172)

Idéntica formación aparece en los casi-hijos ('sobrinos'): confilo(s), confila(s) y confil(es) (para el genérico); y en los casi-padres ('tíos'): conparento(s)/conparenta(s)/conparentes ('tío y tía' conjuntamente), abreviados familiarmente como conpá y conmá. Según esta regla, los abuelos, por tanto, serán grand-parento / grand-parenta (abreviados como grand-pá y grand-má).

Según el modelo de la lengua francesa, construye suegro y suegra como bel-parento y bel-parenta, respectivamente; la misma partícula invariable bel- sirve para formar los equivalentes católicos de cuñado (bel-frato), cuñada (bel-frata), yerno (bel-filo), nuera (bel-fila), yerno у nuera (bel-files); e incluso relaciones más complejas, pero totalmente transparentes, como con-bel-frato, con-bel-frata, con-bel-frates, para designar, respectivamente, concuñado, concuñada, concuñados; o con-bel-parentos, con-bel-parentas, con-bel-parentes para designar consuegros, consuegras y consuegros en conjunto. (Liptay 1890: 174)

Respecto a la aplicación del género a los objetos de uso cotidiano (que, recuérdese, son los vocablos que más modificaciones experimentan en las lenguas, según Liptay), el autor ejemplifica su propuesta analizando los términos sombrero, vestido, zapato y guantes.

Partiendo de que la voz cap es fácilmente identificable en las lenguas occidentales como sinónimo de 'cabeza' (<lat. caput), entiende que el sombrero puede considerarse metonímicamente un abrigo para la cabeza. En este sentido, la voz castellana sombrero no es adecuada para el vocabulario católico, "puesto que indica en general algo que proyecta sombra, que bien podría ser un toldo, techo ó cualquiera otra cosa que sirviera para el mismo objeto" (Liptay 1890: 179). En su lugar sugiere capel(es) como término genérico (<cap-, 'cabeza' y - el 'abrigo) y capelo(s) / capela(s) para designar 
'sombrero de hombre' y 'sombrero de mujer', respectivamente (Liptay 1890: 180), con lo cual, la marca de género se aplica tanto al poseedor (o poseedora) como al objeto poseído, porque es un objeto de uso común en contacto directo con el ser humano (es la única lengua artificial que conozco en la que se establece esta compleja distribución de géneros). Atribuyendo una función derivativa solo a la $-r$ del sufijo -ero / -era (sombrero $>$ sombrerero), el capelor (palabra epicena para Liptay) será el fabricante de capeles ('sombreros'), "sea hombre ó mujer el fabricante" (Liptay 1890: 181); pero si interesa marcar el sexo de quien manufactura se distinguirá entre capeloro y capelora, que podrían traducirse católicamente como fabricanto de sombreros para hombres y fabricanta de sombreros para hombres.

La precisión morfológica puede acentuarse para marcar el sexo del destinatario: si se trata de sombreros para señoras (capelas), el epiceno no puede ser capelor(es), sino capelar(es), término genérico que designa a los fabricantes de sombreros para señoras, sean estos hombres o mujeres; a su vez, se puede distinguir si el fabricante de sombreros para señoras es mujer (capelara) u hombre (capelaro). Por tanto, un capelaro designaría un hombre (o) que fabrica ( $r$ ) abrigos (el) para la cabeza (cap) de una mujer (a). Si este individuo no los manufactura, sino que los vende, será un vendor de capeles (capelos o capelas) o, más sintéticamente, un capel-vendor.

Para designar el oficio de sastre y sus ocupaciones, Liptay recurre a la voz clásica vest ${ }^{21}$ (radical del lat. vestis), reconocible internacionalmente como equivalente de vestido, tanto para el sustantivo como para el verbo; así vesta(s) / vesto(s) designarán respectivamente 'vestido para mujer' / 'vestido para hombre'; vestes, 'vestidos' sin asignación de género; vestuár ('vestuario'); vestor (< lat. vestitor) será el término genérico para sastre, de donde vestoro / vestora ('sastro' / 'sastra', si se me permite la traducción católica del género). Al igual que diferenció en la voz sombrero el sexo del destinatario, distingue entre vestara ('sastra' para señoras) ${ }^{22}$ y vestaro ('sastro' para señores). En la misma línea, el comerciante de ropa será un veste-vendor o un vesto-vendor.

Al no existir una palabra común en las lenguas romances para designar calzado, Liptay propone apelar a la filología comparada, como ya hiciera con sombrero, y buscar una raíz básica sobre la que construir la familia léxica;

21 La razón por la que selecciona esta raíz es singular y se debe a que Liptay considera el oficio de sastre superior a la etimología del nombre que lo designa: en latín (sartor), significa despectivamente 'remendón' (<sarcire, 'coser', 'zurcir, 'reparar'). (Liptay 1890: 182-3)

22 Añade Liptay (1890: 183): “vestara ('sastra' para señoras) [...] sastra de oficio y no por ser la mujer del sastre". 
dicha raíz es ped (o pied) 'pie' y sobre ella forma piedo(s) ('pie de hombre') / pieda(s) ('pie de mujer'), reservando piedes (o pedes) para el plural genérico (Liptay 1890: 185). Como ya disponía de la raíz vest- ('vestido', 'abrigo'), construye piedo-vest ('calzado masculino') / pieda-vest (calzado femenino), pues el calzado es, metonímicamente, 'un abrigo para el pie'; el zapatero, 'el hacedor de tal abrigo', se designará con los términos piedovestor ('fabricante de calzado para hombres') y pieda-vestor ('fabricante de calzado para mujeres'); para mayor precisión, añade una $-o$ para indicar que el agente que manufactura tanto calzado de hombre como de mujer es masculino (piedo-vestoro/ pieda-vestoro); y una - a para indicar que es femenino: piedo-vestora/ pieda-vestora.

Una vez establecidos los términos católicos para calzado, ha de encontrar un vocablo más preciso para designar zapato. Al igual que en la familia léxica de sombrero utilizó un sufijo-el / - al con el significado de 'abrigo', ahora forma zapato de manera similar: piedal o pedal; de donde pedalo ('zapato de hombre') /pedala ('zapato de mujer'). A partir de esta distribución crea pedalin (designación genérica para zapato plano), pedalino ('zapato plano masculino'), pedalina ('zapato plano femenino') y pedalon ('bota'). Los términos que designan 'oficio' o 'profesión' serían: pedalor ('zapatero') / pedalar ('zapatera'), si no se especifica el sexo del destinatario del producto; frente a pedaloro ('zapatero empleado en la fabricación de zapatos para hombre') /pedalora ('zapatera empleada en la fabricación de zapatos para hombre'), si se marca el género del destinatario/a. El vendedor de zapatos se designaría como pedal-vendor (pedal-vendora, en femenino). (Liptay 1890: 185-7)

El último objeto de uso cotidiano que trata Liptay son los guantes, cuya denominación en la lengua católica parte de la forma latina digitalia ( $<$ lat. tegumenta digitalia 'abrigos para los dedos'); así, digital(es) significa 'guante(s)' sin especificar el poseedor, frente a digitalos ('guantes de hombre') y digitalas ('guantes de mujer'). De la raíz de esta voz parte Liptay para proponer digit ('dedo') y de ahí, digito(s)/digita(s), que designan 'dedos de hombre' y 'dedos de mujer', respectivamente, mientras que digites significa 'dedos en general'. El diminutivo, digitalin, puede emplearse como equivalente de 'dedal'. (Liptay 1890: 189) 


\section{CONCLUSIONES}

Las confluencias ideológicas y las contradicciones que impregnan el proyecto de la lengua católica son muy potentes. Para un autor como Liptay, casi apátrida, la lengua católica podía convertirse en una especie de hogar lingüístico; de ahí que aprovechara la eclosión lingüística del siglo XIX para diseñar una lengua paneuropea, que no es sino el descubrimiento de un poso latino evolucionado que pervive en la diversidad lingüística occidental. A su vez, esta lengua paneuropea -menos arbitraria que otros proyectos mixtos, como el volapük, y más racional y común que otros diseños basados en el latín simplificado-era una oportunidad inmejorable para llevar a la práctica la ortografía fonética que defendía el movimiento chileno de reforma ortográfica; entre ellos, Lenz, con quien mantenía una sólida amistad. Liptay incorporó en su lengua católica muchas de las recomendaciones ortográficas de Bello, pero nunca desdeñó recurrir a la etimología clásica para la formación racional de palabras. De hecho, su postura es muy ambigua. El único texto que redacta ateniéndose a los principios de la ortografía fonética es su intervención en el I Congreso Científico de Valparaíso. Sovre la Vi la B en castellano (1893), donde escribe:

[...] impulsados por sus sentimientos del altruismo mas jeneroso, estos reformadores à outrance no toman en cuenta el savio consejo de los antiguos festina lente, ni el adajio de los modernos chi va piano va lontano cuando tratan, de un solo golpe autoritario, acer triunfar la razon sovre la rutina, $\mathrm{o}$, mejor dicho, su teoria o version de la verdad sobre la practica inveterada de las tradiciones seculares. Para evitar [...] un antagonismo tan formidable, me decidí por la evolucion en preferencia a la revolucion ortografica. (Liptay 1893: 7-8)

Aun así, es aventurado determinar si estaba realmente convencido de que la lengua católica -"idioma común de los pueblos civilizados"-podría servir como puente ideológico que abriera el continente americano a la modernidad europea o, por el contrario, si la utilizó en beneficio propio para ganarse el respeto intelectual y el mecenazgo de sus contemporáneos.

Por último, la racionalización extrema del género católico no tiene precedentes, tampoco continuadores, en la historia de las lenguas artificiales. Lo interesante de su propuesta es que el género, que comienza en la esfera nocional (solo se atribuye a entidades sexuadas), acaba convertido en un asunto de concordancia extrema (recuérdense los ejemplos analizados de sombrero, vestido, zapato y guantes), aunque esta concordancia encuentre su razón y acomodo en la esfera extralingüística. Insistir en que las mujeres 
deben estar representadas en la gramática (vestara significa 'sastra de oficio' y no 'la mujer del sastre') resulta una extraordinaria novedad, quizá justificada desde su neomaltusianismo feminista, y solo comparable con las lenguas alternativas para mujeres (también diseñadas por mujeres) que se proponen en algunos relatos utópicos de la ciencia ficción moderna.

\section{REFERENCIAS BIBLIOGRÁFICAS}

Alvar EzQuerra, Manuel. 2019. Biblioteca Virtual de la Filología Española (BVFE): directorio bibliográfico de gramáticas, diccionarios, obras de ortografia, ortología, prosodia, métrica, diálogos e historia de la lengua [en línea]. Disponible en www.bvfe. es [Consulta 23/07/2019].

Bello, Andrés. 1884. Obras Completas. Volumen 5. Opúsculos gramaticales. Santiago de Chile: Pedro G. Ramírez [en línea]. Disponible en https://archive.org/stream/ opusculosgramati00belluoft\#page/n6/mode/2up[Consulta 23/07/2019].

Benot, Eduardo. 1892. Memoria. Actas del Congreso Literario Hispano-Americano. IV Centenario del Descubrimiento de América. Sección Primera. Filología, pp. 257-261. [en línea]. Disponible en http://cvc.cervantes.es/lengua/congreso_literario/pdf/CVC_ congreso_257.pdf [Consulta 23/07/2019].

Brugmann, KARL y August LeSKIEn. 1907. Zur Kritik der künstlichen Weltsprachen. Strassburg: Trübner. [en línea]. Disponible en https://archive.org/details/zurkritikderkn00bruguoft/ page/n2 [Consulta 23/07/2019].

CÁceres, Valentina y Darío Rojas. 2019. Rodolfo Lenz y la reforma ortográfica chilena: ciencia, tradición y política del lenguaje. Boletín de Filología LIV/1: 65-93.

Calero Vaquera, M. a Luisa. 1996. Un proyecto español anónimo (1852) de lengua universal. Revista de Filología Española 76: 91-113.

2010. Las irregularidades lingüísticas desde la perspectiva de los inventores de lenguas universales. En Carsten Sinner y Alfonso Zamorano (eds.). La excepción en la gramática española. Perspectivas de análisis, pp. 17-36. Madrid / Frankfurt: Iberoamericana / Vervuert.

Couturat, Louis y LÉOPOLd LÉAu. 1903. Histoire de la langue universelle. Hildesheim: Georg Olms Verlag.

Galán Rodríguez, Carmen. 2012. Lenguas artificiales. En Alfonso Zamorano (coord.). Reflexión Lingüística y Lengua en la España del XIX. Marcos, panoramas y nuevas aportaciones, pp. 417-442. München: LINCOM.

2016. En los márgenes del lenguaje: los outsiders de la Lingüística. En Gerda Haßler y María Luisa Calero Vaquera (eds.). La Historiografía de la lingüística y la memoria de la lingüistica moderna, pp. 57-83. Münster: Nodus Publikationen.

García del Río, JuAn y ANDRÉs Bello. 1823. Indicaciones sobre la conveniencia de simplificar i unificar la ortografía en América. Caracas: Imprenta de Domingo Navas.

Garner, Samuel. 1894. The Problem of an International Language. Review: Langue Catholique. Projet d'un idioma international sans construction grammaticale by Alberto Liptay, . Modern Language Notes IX: 3: 86-91 [en línea]. Disponible en www.jstor.org/ stable/2918559 [Consulta 27/07/2019] 
GouÉDART, JEAN-JACQUes. 1993. Le Docteur Alberto de Liptay (1859-1922), néo-malthusien. Un curieux ancêtre de l'I.V.G. et du planning familial. Tesis doctoral inédita, leída en la Universidad de Caen ( Normandía).

Hassler, Gerda. 2017. La semiótica al inicio del siglo XX: condiciones de un cambio en la metodología lingüística. Boletín de la Sociedad Española de Historiografia Lingüistica 11: $105-121$.

Hassler, Gerda y María Luisa Calero Vaquera (eds.). 2016. La Historiografía de la lingüística y la memoria de la lingüistica moderna. Münster: Nodus Publikationen.

Hello, Eric. 2016. Les néomalthusiens français et les sciences biomédicales (1880-1940). Tesis Doctoral inédita, Université de Bordeaux.

Jespersen, Otto. 1894. Progress in Language with Speciel Reference to English. London: Sommendheim.

La CIUDAd de Dios. Revista Religiosa, Cientifica Y literaria. Sección Bibliográfica. Madrid: Real Monasterio de San Lorenzo del Escorial, 1890, vol. XXIII, pp. 607-608 [en línea]. Disponible en https://www.yumpu.com/es/document/read/18880096/la-ciudad-dedios/611 [Consulta 01/07/2019]

LA FARMACIA ESPAÑOLA. Revista científica y profesional. Periódico oficial del Colegio de Farmacéuticos de Madrid. Año XXIL, Madrid, 2 de octubre de 1890, 40: 633.

LA ILUSTRACIÓN ESPAÑOLA Y AMERICANA. Año IV, 30 de enero de 1894.

Liptay, Alberto. 1888. El darwinismo. ¿Cuál es la posición del hombre en el Universo? Valparaíso: Imprenta Librería El Mercurio.

1890. La lengua católica; ó sea, Proyecto de un idioma internacional sin construcción gramatical [por el] dr. Alberto Liptay. Paris: A. Roger y F. Chernoviz.

1891. Eine Gemeinsprache der Kulturvölker. Leipzig: Brockhaus.

1892. Langue catholique. Projet d'un idiome international sans construction grammaticale. Paris: Bouillon.

1893. Sovre la posibilidad de un idioma internacional obtenido por sufragio universal. Actas I Congreso Científico de Valparaíso. Sovre la Vi la B en castellano, pp. 75-103. Santiago de Chile: Imprenta Cervantes..

1897. La possibilité d'un idioma international sans grammaire. Paris: Edición del autor.

1903. Le mystère posthume; causeries médicales sur la mort \& la survie. Paris: Edición del autor.

1904. La vie sexuelle des monstres, avec mille et une observations curieuses sur leurs organes génitaux. Paris: Edición del autor.

1906. La préservation sexuelle. Paris: Edición del autor.

1907. Bréviaire de la femme enceinte. Paris.

1908. Prophylaxia sexualis; causeries médicales sur la préservation et les préservatifs sexuels: d'après les données les plus récentes de la science française et étrangère. Paris.

1911. Pour et contre Malthus: la préservation sexuelle, la prolétarisation nationale, la Sainte Inquisition bérengiste, la liberté de la presse existe-t-elle sous la troisième République libertaire, égalitaire et fraternitaire, Paris.

Lotт, Julius. 1890. Un Lingua Internazional: Grammatika et Vokabular Pro Angleses, Germanes, Romanes et Pro Cultivates de Tut Mond. Leipzig: Frankenstein \& Wagner.

O’Ryan, Pedro. 1886. Memoria del Cirujano Mayor del Departamento. Memoria del Ministro de Marina al Congreso Nacional. Santiago de Chile: Imprenta Nacional. 89-94 [en línea]. Disponible en https://archive.org/stream/memoriachilemin00unkngoog\#page/n0/ mode/2up. [Consulta 20/07/2019]. 
PAYÀs, GERTRUDIs. 2008. Tradukzión i rrebelión ortográfika. TRANS: Revista de traductología. 12: $15-28$.

Pei, Mario. 1958. One language for the world. NewYork: Biblio-Moser.

Ronsin, Francis. 1980. La Grève des ventres. Propagande néo-malthusienne et baise de la natalité en France (XIX-XX siècles). Paris: Aubier Montaigne.

Sagredo Baeza, Rafael (ed.). 2010. Ciencia-Mundo. Orden republicano, arte y nación en América. Santiago de Chile: Editorial Universitaria.

Salomón Gebhard, José Luis. 2005. Ficciones de la Lengua Universal. Revista Electrónica: Documentos Lingüisticos y Literarios UACh. 28: 89-93 [en línea]. Disponible en http:// www.humanidades.uach.cl/documentos_linguisticos/document.php?id=99 [Consulta 03/07/2019].

Sinner, Carsten y Alfonso Zamorano Aguilar (eds.). La excepción en la gramática española. Perspectivas de análisis. Madrid / Frankfurt am Main: Iberoamericana / Vervuert.

Velarde Lombraña, Julián. 1987. Proyectos de lengua universal ideados por españoles. Taula. Quaderns de Pensament 7/8: 7-78. 27/1: 59-77.

Zamorano Aguilar, Alfonso (coord.). Reflexión Lingüística y Lengua en la España del XIX. Marcos, panoramas y nuevas aportaciones. München: LINCOM. 